\title{
Pobreza y Estrategias Familiares: Debates y Reflexiones
}

\section{Catalina Arteaga A.}

Dra. (C) en Ciencias Políticas y Sociales, mención Sociología. UNAM - México Profesora Departamento de Sociología Universidad de Chile.

carteaga@uchile.cl

Resumen

El presente artículo busca sistematizar críticamente algunos estudios acerca de las estrategias familiares y proponer un ordenamiento en enfoques teóricometodológicos, a partir de las dimensiones centrales que los trabajos utilizan para el análisis.

Abstract

This article seeks to critically systematize some studies on family strategies in order to propose a theoretical - methodological approach, from the central dimensions used for analysis.

Palabras Clave: Pobreza, Estrategias, Familia, Metodologías, Teorías

\section{I ntroducción}

El estudio de las estrategias familiares cobró gran relevancia en la región en los años ochenta, a partir de la implementación de las políticas de ajuste estructural que se desarrollaron en distintos países ${ }^{1}$. Se desarrolló un interés por analizar los efectos de dichas políticas en los niveles de pobreza y en las acciones desarrolladas por las familias para su reproducción. Si bien el marco de las estrategias ha sido criticado, lo cierto es que siguen desarrollándose estudios en torno a las actividades que desarrollan las familias de sectores más pobres para su sobrevivencia, en un marco en el cual el fenómeno de la pobreza y la vulnerabilidad en la región - a pesar de su disminución-, sigue siendo un fenómeno de importancia ${ }^{2}$, insertos en procesos de diferenciación social y transformaciones económicas y socioculturales que imprimen nuevas interrogantes en la solución de dicha temática.

Si analizamos parte de la producción de América Latina, se encuentran estudios de distintos países -Chile, México, Uruguay- que con diversos enfoques metodológicos abordaron el tema de las estrategias familiares con relativa profundidad; dichos estudios se realizaron en ámbitos principalmente urbanos y en sectores generalmente pobres, siendo pioneros los estudios de Raczinsky en Chile en los años setenta.

La pregunta que subyacía a estos trabajos, era acerca de las diversas formas en que las familias hacían frente a las difíciles condiciones de vida. A partir de dichos trabajos se conocieron distintas estrategias que los hogares han realizado para enfrentar 
situaciones difíciles para reproducirse y sobrevivir, entre ellas encontramos: intensificación del trabajo asalariado y doméstico; reacomodo en la división del trabajo; ampliación de los vínculos sociales; cambios en el tamaño y composición del hogar; migraciones; transformaciones en los patrones de consumo -entre otros aspectos-.

A partir de la revisión de algunos de estos estudios, planteamos un ordenamiento en cuatro enfoques a partir de las variables y dimensiones centrales que los autores analizan como explicativas de los comportamientos familiares: enfoque de estrategias de sobrevivencia, que acentúa el análisis de la estructura, composición y ciclo doméstico del hogar; enfoque de redes, -que ha priorizado en la capacidad de los grupos domésticos para articularse y sobrevivir-; el enfoque de vulnerabilidad-activosestructura de oportunidades, el cual ha centrado el análisis en el tema de los recursos y activos de los hogares y la potenciación o limitación de su desarrollo a partir de las características de la comunidad y la normatividad (Moser, 1996, 1998), así como la relación con la estructura de oportunidades (Kaztman y otros, 1999, 2002). Por su parte el enfoque del curso de vida, el que ha puesto el acento en las estrategias familiares en un contexto de cambio sociocultural e institucional (Hareven, 2000; Moen y Wethington, 1992).

A pesar del rechazo de algunos autores a continuar con el estudio de las estrategias, lo cierto es que en diferentes trabajos acerca de la pobreza, la vulnerabilidad, la reproducción de los hogares y la implementación de políticas sociales, el problema de las estrategias familiares, es decir, de las distintas actividades y comportamientos que las familias realizan para su reproducción, sigue siendo importante para entender los mecanismos que operan al interior de los hogares para mantener o aumentar su bienestar o para no caer en mayores niveles de pobreza. Ello tiene relevancia en la medida que puede incidir en conocimientos y recomendaciones para la elaboración de políticas destinadas a disminuir la pobreza.

A pesar de los avances e importantes aportes en los estudios sobre estrategias, ha habido algunas críticas, limitaciones y vacíos que es importante asumir e intentar avanzar en ese sentido. En términos sintéticos, pueden señalarse: la estrecha relación entre el concepto de "estrategia" y su vinculación al paradigma de la elección racional; la visión idealizada de las familias en condiciones de pobreza desde la perspectiva de algunos estudios; la incapacidad de este enfoque de analizar el conflicto doméstico. En cuanto a los vacíos, pensamos que se ha explorado poco acerca de la estructura de oportunidades; los valores y motivaciones individuales; la relación de los individuos y las familias con las instituciones que pueden limitar o incentivar cierto tipo de estrategias.

Además de estas consideraciones, es importante señalar que el contexto en que se han estudiado las estrategias ha ido variando en el tiempo. En América Latina se han producido cambios importantes en el contexto sociocultural, económico e institucional que implican transformaciones en las formas de vida de la población, las prácticas económicas, las formas de organización social y política, la constitución de las identidades, la dinámica familiar y las percepciones de hombres y mujeres frente a su entorno. Un ejemplo de ello son los cambios en el ámbito del trabajo y de los factores de movilidad laboral, que llevan a algunos autores a plantear que si bien la movilidad en este ámbito se vinculaba a las condiciones económicas y el nivel educativo, hoy en 
día una buena educación no necesariamente posibilita un mejor empleo. Otros ámbitos de cambio se relacionan con las dinámicas demográficas, en cuanto a la estructura y composición de los hogares y a las relaciones entre géneros y generaciones al interior de los mismos. ${ }^{3}$

Dichos fenómenos implican alteraciones en los referentes de constitución de las identidades individuales y colectivas y asimismo en las aspiraciones y proyectos de vida de los sujetos. Como han señalado algunos autores, en la época actual las identidades tienen rasgos de flexibilidad, apertura e indeterminación antes desconocidos.

\section{De la racionalidad de las estrategias}

Basándose en los diversos paradigmas que analizan la acción social, en general es posible ubicar un concepto de estrategia dentro del enfoque de la acción racional, que subyace a algunos estudios desarrollados. Al respecto es posible aludir a un uso amplio y uno restringido del mismo. En términos generales - desde esta perspectiva-, por estrategia es posible entender “...toda selección de cursos alternativos de acción (recursos tácticos) por su virtualidad para producir resultados futuros (objetivos estratégicos) en situaciones de incertidumbre." (Garrido y Gil, 1993).

Esta concepción delimita tres requisitos necesarios para el desarrollo de estrategias: la existencia de un margen de maniobra o un abanico de posibilidades; la existencia de objetivos previamente definidos y la presencia de alguna clase de incertidumbre en el entorno (Garrido y Gil, 1993). En este sentido, como se advierte, dicho concepto se acerca a la idea de acción racional.

Aplicado a la familia, el concepto de estrategias familiares desde esta perspectiva se puede definir como "...aquellas asignaciones de recursos humanos y materiales a actividades relacionadas entre sí por parentesco...con el objeto de maximizar su aptitud para adaptarse a entornos materiales y sociales.". Los recursos tácticos de las estrategias son los que pueden activar los miembros de las familias; los bienes que controlan y las actividades que pueden realizar. Los objetivos familiares pueden entenderse en términos generales como la búsqueda de un mayor y mejor éxito (Garrido y Gil, 1993). En esta concepción, la noción de estrategia familiar se centra en el margen de variación de los recursos tácticos que dispone la familia y no tanto en sus objetivos estratégicos y la naturaleza de sus relaciones con las demás familias; la estrategia de la familia depende de la elasticidad con que éstas adaptan su comportamiento al entorno (Garrido y Gil, 1993).

En este sentido, el concepto de estrategia aplicado a las familias deriva de un predominio teórico del paradigma económico, el cual al analizar a esta institución y su reproducción observa sus acciones en términos estratégicos. Desde esta visión, las acciones llevadas a cabo por los hogares para su reproducción son analizadas desde la óptica individualista y racional como resultado de un proceso de decisión autónomo, racional e independiente de otras variables. Desde esta perspectiva tanto los objetivos como los medios de estas unidades implican asignación de recursos en términos generacionales. 
Los recursos privilegiados desde esta noción, generalmente aluden a una dimensión económica de los hogares. Desde esta perspectiva, Meert define las estrategias como “... las intenciones de los hogares individuales para superar los obstáculos macrosociales que obstruyen sus intenciones y objetivos; en esta interpretación las estrategias tienen claramente una dimensión económica y están motivadas por los hogares para ahorrar dinero en la esfera del consumo; ganar dinero extra en la esfera productiva o para cambiar acciones mercantiles por acciones no monetaristas para sobrevivir." (Meert, 2000).

Dichas estrategias además permitirían a los hogares integrarse en diferentes modalidades al sistema económico que produce los recursos necesarios para vivir. Así, el autor retoma el concepto de "modos de integración económica" de Polanyi, para referirse a las formas en que dichos hogares se pueden vincular, detectando tres modalidades: intercambio mercantil; redistribución y reciprocidad. De acuerdo a su estudio, en contextos urbanos, las estrategias que permiten la integración económica a través del mercado y redistribución son más relevantes que las de reciprocidad, que aumentan en importancia en contextos rurales. En estos hogares, la medida en que pueden desarrollar estrategias, depende de sus redes sociales y de las relaciones que pueden desarrollar a partir de esas redes (Meert, 2000:327).

Frente a esta concepción de estrategias, pueden recogerse y realizarse un sinnúmero de críticas al concepto - a las cuales podemos agregar algunas-, las cuales derivan por una parte de aquellas que se formulan para cuestionar el modelo de acción racional y, por otra, de la aplicación de dicho concepto a la familia:

- El modelo de acción racional explica a los individuos como libres y racionales, con un gran sesgo ideológico. Parte de supuestos ideales, como la estabilidad y perdurabilidad de las preferencias, sin tomar en cuenta la posibilidad de cambio en el tiempo e inestabilidad de las mismas, lo que haría cuestionar el modelo (Elster). No se trata de que la conducta humana sea única y exclusivamente racional, sino que metodológicamente se presupone ésta y se plantea analizarla como si lo fuera, para explicar con pocos elementos la acción humana y predecir sus resultados.

- Se sabe muy poco de las preferencias individuales y sólo se obtiene información de ellas a través de las elecciones. Sobre los motivos que las configuran no se sabe nada, es decir, el concepto de preferencia es informativamente muy limitado.

- Por otra parte, es imposible que la gente tenga toda la información; la búsqueda de información óptima colapsaría a menudo la elección. En la mayoría de las elecciones, se busca satisfacer el interés más que maximizarlo.

- Otra de las críticas a la teoría de elección racional es la imposibilidad de dicho planteamiento de dar cuenta de la multiplicidad de elementos que inciden en la conducta y en la toma de decisiones, destacando por ejemplo las motivaciones y creencias.

Las críticas más específicas a esta noción racional de las estrategias familiares, son:

-El concepto de estrategia familiar implica la posibilidad de decisión por parte de las familias, frente a una multiplicidad de opciones. Dicho planteamiento es cuestionado 
en tanto existen múltiples limitantes a las posibilidades de decisión, que se ubican en el entorno familiar - el contexto- como en las características mismas de estructura y composición del hogar.

- La noción de estrategias familiares, al estar vinculada al paradigma económico, ha priorizado el análisis de la reproducción económica de los hogares, dejando de lado las otras formas de reproducción (biológica, sociocultural).

-El concepto de estrategias familiares en cierta medida ha obviado la presencia del conflicto y la diferenciación al interior de los hogares. Las estrategias familiares pueden en ocasiones oponerse o contradecirse con las estrategias individuales de sus miembros. Diversos estudios, particularmente aquellos desarrollados desde el enfoque de género, han cuestionado la igualdad en las posibilidades de acción y decisión por parte de los distintos integrantes de los grupos domésticos.

-En términos teórico-metodológicos, la noción de estrategias familiares supone un problema con respecto al nivel de agregación relacionado con lo señalado anteriormente. La observación de las estrategias familiares puede darse a partir del análisis particular de las decisiones de sus miembros o darse con base en las acciones colectivas desarrolladas por la familia.

- Al plantear la conciencia de las intenciones que llevan a las familias a articular estrategias, se dan al menos tres problemas: muchas veces la acción familiar carece de intenciones; a veces los resultados más estratégicos de las familias son subproductos colaterales; por otra parte, las intenciones conscientes de la acción familiar pueden ser contradictorias, cambiantes e inciertas (Garrido y Gil, 1993).

- Otra crítica que se puede hacer a la noción de estrategia familiar es el excesivo enfoque en el individuo, sin considerar de manera adecuada las limitaciones del entorno al comportamiento individual.

\section{Propuesta de enfoques}

A partir de la revisión de distintos estudios realizados principalmente en Latinoamérica con respecto al tema de las estrategias familiares, se ha optado por agrupar dichos trabajos en cuatro grandes enfoques de acuerdo a las dimensiones centrales que nos parece que los autores analizan como explicativas para el desarrollo de los comportamientos familiares: a) enfoque de estrategias de sobrevivencia, b) enfoque de redes, c) enfoque de vulnerabilidad-activos-estructura de oportunidades; d) enfoque de curso de vida. Ello no implica que los estudios situados en alguno de los enfoques no consideren las otras dimensiones, sin embargo, dan más centralidad a ciertas variables a la hora del análisis.

En términos sintéticos se puede decir que el primer enfoque ha dado mayor importancia a la variable de estructura, composición y ciclo doméstico del hogar como elementos centrales en la relación que se establece entre la familia y las estrategias fundamentalmente laborales, priorizando por el análisis de la vinculación entre el hogar y el mercado de trabajo (González de la Rocha, 1986; Selby et. Al., 1990, 1994; García y Oliveira, 1994; Cortés y Rubalcava, 1991). Por su parte, el enfoque de redes ha priorizado en la capacidad de los grupos domésticos de articularse para sobrevivir 
(Lomnitz, 1994; Espinoza, 1995). El enfoque de vulnerabilidad-activos-estructura de oportunidades ha centrado el análisis en el tema de los recursos y activos de los hogares y la potenciación o limitación de su desarrollo a partir de las características de la comunidad y la normatividad (Moser, 1996, 1998), así como la relación con la estructura de oportunidades (Kaztman y otros, 1999, 2002). Por su parte el enfoque del curso de vida ha puesto el acento en las estrategias familiares en un contexto de cambio sociocultural e institucional (Hareven, 2000; Moen y Wethington, 1992).

a) Enfoque de las estrategias de sobrevivencia

El enfoque de las estrategias de sobrevivencia ${ }^{4}$ fue desarrollado en Latinoamérica desde fines de los años setenta ${ }^{5}$, a partir del cuestionamiento sobre las formas en que las familias de sectores pobres urbanos hacían frente a las difíciles condiciones económicas del período.

Como señala González de la Rocha, se trataba de dar respuesta y criticar la idea de que los pobres urbanos tenían un carácter marginal y desorganizado, se intentaba dar cuenta de las formas en que efectivamente dichas familias enfrentaban a través de diversos mecanismos su vivir cotidiano. No obstante, como plantea la autora, se enfatizó en la racionalidad y capacidad de adaptación de los grupos domésticos, sin ahondar en el conflicto doméstico y en la relación de los grupos con el entorno laboral.

En ese sentido estudiar las estrategias buscaba lo que actualmente siguen señalando estudios como los de Moser: abrir la caja negra de los grupos domésticos y desentrañar las formas en que éstos llevan a cabo su sobrevivencia cotidiana. Se trataba de “...describir conductas y, por ensayo y error, ir proponiendo hipótesis acerca de los principios que organizan esas conductas aparentemente dispersas, sus propias formas de racionalidad, su lógica, para luego someterlas a prueba y explicarlas..." (Barsotti, 1981: 184).

La mayor parte de los estudios desarrollados bajo este enfoque se centró en la reproducción económica de los hogares de sectores marginales urbanos (Duque y Pastrana, 1973; Lomnitz, 1975) y las formas en que éstos se articulaban con el mercado de trabajo. Con base en este enfoque, algunos autores se refirieron a un concepto más amplio: "estrategias familiares de vida", para orientar el estudio no sólo a la reproducción económica de los hogares, sino también a la reproducción biológica; dicho cambio conceptual además involucraba no sólo a los grupos subordinados, sino a todos los grupos sociales, por tanto hacía referencia a un fenómeno social más global (Argüello, 1981:193). No obstante, esta última propuesta no fue muy desarrollada y se siguió enfatizando en el estudio del comportamiento de familias pobres urbanas.

Los estudios sobre estrategias familiares retomaron fuerza en la década de los ochenta a partir del ajuste estructural que impactó a los distintos países de Latinoamérica. Las formas en que las familias de sectores urbanos hicieron frente a estos fenómenos y las crisis económicas recurrentes, fueron estudiadas bajo este enfoque. La diversidad de metodologías desarrolladas nos hace diferenciar a grandes rasgos entre estudios de tipo cualitativo - principalmente antropológicos- y los de tipo cuantitativo. La revisión se centra principalmente en México, aunque también se revisaron trabajos de otros países. 


\section{Estudios cualitativos}

El estudio de las estrategias desarrolladas por los hogares en México, ha estado vinculado en gran medida al impacto que ha tenido el proceso de ajuste estructural, principalmente en aquellos de bajos ingresos.

Una parte de estos estudios se han orientado desde una perspectiva antropológica. En ésta se incluyen algunos trabajos realizados por Mercedes González de la Rocha en la ciudad de Guadalajara (1986, 1990, 1991), cuyo objetivo fue analizar el modo en que se vio afectada la unidad doméstica en su estructura y organización internas y en sus relaciones con el mercado de trabajo. En dicho análisis fue importante la observación de las estrategias gestadas al interior del territorio doméstico, como resultado del control que los miembros de dicho grupo tienen sobre los recursos internos, aunque dichas estrategias se encuentran condicionadas por el contexto externo, por las condiciones económicas más amplias, por el mercado de trabajo (González de la Rocha, 1986.).

A partir de dichos trabajos, se pueden delimitar algunas estrategias observadas por la autora que desarrollan los hogares en épocas de dificultades económicas y que posteriormente han sido reforzadas con los hallazgos de otros estudios. ${ }^{6}$ Las estrategias que se plantean, dicen relación con:

a) Intensificación del trabajo asalariado y doméstico. Esta estrategia supone la incorporación de un número mayor de miembros en el mercado de trabajo y la concentración de actividades domésticas en las manos de los miembros dedicados a éstas.

b) Reacomodo en la división del trabajo. Esta situación se da a través de la creciente participación de las mujeres de más de catorce años y de los hombres menores de quince años en la actividad económica remunerada.

c) Cambios en el tamaño y composición del hogar. La autora señala que en su estudio, los grupos domésticos crecen sobretodo por la incorporación de miembros en edad de trabajar y la retención de los integrantes hábiles; aunque también señala que han crecido por vías naturales a partir del desarrollo del ciclo doméstico. No obstante, el aumento se habría dado principalmente a partir de la incorporación de miembros capaces de generar ingresos.

d) Cambios en la estructura social del hogar. Esta situación deriva del incremento de hogares extensos y múltiples y la disminución de hogares nucleares.

e) Modificación en los patrones de consumo. Aunque señala que éste había sido protegido con relativo éxito, en familias de bajos recursos se observó una disminución de algunos productos (carne, pescado, lácteos) y el aumento de otros (leche Liconsa, huevos, tortillas, cereales y vísceras), (González de la Rocha, 1990).

Por su parte, Selby et. Al. (1990, 1994), con base en un estudio realizado en hogares urbanos a nivel nacional en 1977-1979 y en la ciudad de Oaxaca en 1988, coincide en señalar algunas de las estrategias desarrolladas por los estudios arriba citados.

Plantea como parte de las estrategias desarrolladas por los hogares para enfrentar las crisis económicas, tres cambios evidentes a nivel familiar: un descenso de la tasa de natalidad (de 2.9 a 2.3); una disminución de los miembros del hogar (de 5.3 a 4.6); la 
complejización de los hogares, es decir, la intensificación de la variedad de formas familiares. Señala que a medida que aumenta el ingreso familiar, aumenta el número de miembros del hogar, el número de participantes en la fuerza de trabajo, la proporción de hogares sustentados en familias extensas y la variedad de formas familiares.

Por su parte G. Andrea Cornia, en un trabajo publicado en 1987 dentro de un conjunto de estudios relativos al impacto del ajuste estructural en términos sociales, agrupa las estrategias de sobrevivencia en tres categorías, a partir de la sistematización de estudios realizados principalmente en países de América Latina. Al respecto señala:

Estrategias destinadas a la generación de recursos. El objetivo de éstas es el intento de mantener el nivel de ingresos corrientes de la familia o contener su descenso. Dicha estrategia se articula a partir de diversas formas:

- Incremento de la oferta de fuerza de trabajo en la economía. Dicho incremento de acuerdo a distintos estudios se habría concentrado en mujeres y se habría dado a partir del aumento de empleos de tipo informal.

-Incremento de la producción propia. Dicha tendencia se da sobretodo en la producción alimenticia, la construcción de alojamientos y en algunos servicios.

- Cambios en la posición de activos-pasivos. Esto implica el endeudamiento del consumidor con las tiendas del barrio o en caso extremo, la venta de activos.

-Aumento del flujo de ingresos por transferencia. Lo que implica transferencias privadas que pueden darse dentro de la propia familia; o transferencias públicas (subsidios, subvenciones, comidas escolares).

Estrategias para mejorar la eficacia de los recursos existentes. Estas estrategias se encuentran orientadas a moderar el descenso de los niveles de consumo material y de bienestar por una disminución generalizada de recursos.

- Cambios en los hábitos de compra. Ello implica cambiar el tipo artículos para la alimentación, por ejemplo, en el tipo, cantidad o temporalidad.

- Cambios en los hábitos de preparación de alimentos. Una de sus expresiones es la organización de comedores populares en distintos países de América Latina durante la crisis de los ochenta.

- Cambios en las pautas globales de consumo. Esto puede llevar a eliminar del consumo artículos considerados no básicos, como vestimenta; y luego recortes de artículos básicos relacionados con agua, combustible, alimentación.

- Cambios en las pautas dietéticas. Ello puede llevar a una disminución de la calidad y equilibrio de las dietas y una reducción del consumo de alimentos.

- Cambios en la distribución intra-familiar de alimentos. Esto puede darse en algunos casos, aunque la autora no aporta datos contundentes al respecto, pero se refiere a 
que las mujeres y jóvenes serían mayormente desfavorecidos dentro de la familia en cuanto a la distribución de alimentos.

\section{Estrategias de familia extendida y de migración}

Dichas estrategias implican un cambio en la composición y localización de la familia para aumentar su potencial de ingresos o cambiar la relación entre necesidades y recursos.

- Cambios en la composición y organización de la familia. Esto implica bien la incorporación de nuevos miembros para enfrentar los gastos; o traspasando alguno de sus miembros a otras familias.

- Emigración. También es una estrategia que desarrollan los hogares para enfrentar las difíciles condiciones de vida y trabajo.

\section{Estudios cuantitativos}

Desde una perspectiva macro, se han desarrollado estudios que analizan datos a nivel nacional o en ciudades específicas para determinar las estrategias desarrolladas por los hogares mexicanos en la época del ajuste.

Cortés y Rubalcava (1991, en Damián 2002) analizan las encuestas nacionales de ingreso y gasto de los hogares y señalan que los hogares entre 1977 y 1984 utilizaron la estrategia del envío de un mayor número de miembros al mercado laboral, principalmente a mujeres, jóvenes y niños dependiendo del tamaño de la familia, su composición por sexo, la etapa del ciclo doméstico, el tipo de familia y las oportunidades dadas por el entorno.

Dentro de esos estudios, Tuirán (1993), con base en la categorización de las estrategias de Cornia señaladas, analiza una encuesta sobre ingreso y consumo familiar desarrollada por el Instituto Nacional del Consumidor, señalando la evolución del gasto e ingresos familiares en un periodo de tres años, destacando estrategias de obtención de recursos; mejora de la eficacia y cambios en la composición y organización familiar.

Por su parte, García y Oliveira (1994) en su estudio sobre trabajo femenino y vida familiar, señalan la intensificación por parte de las familias de diversos mecanismos de reproducción cotidiana frente a situaciones económicas difíciles, particularmente el trabajo extradoméstico de mujeres de sectores populares para la obtención de recursos monetarios. A partir del análisis de encuestas de fecundidad señalan un cambio en la participación económica femenina de mujeres jóvenes sin hijos hasta principios de los setenta, a un incremento de mujeres de 20 a 49 años desde 1976 a 1987 (último año de su análisis) y señalan que a pesar de los problemas de comparabilidad de la información, se da un evidente aumento de la tasa de participación femenina en el periodo 1970-1987, con la mayor presencia de mujeres de más de 25 años.

Más recientemente, Damián (2002), a partir de una revisión crítica de algunos estudios sobre estrategias laborales de sobrevivencia, cuestiona el planteo de que un 
mayor número de miembros del hogar ingresó al mercado de trabajo durante el periodo del ajuste. Con base en el análisis de información proveniente de las encuestas de empleo, señala que a pesar de que el número de trabajadores aumentó en México, los hogares sufrieron un grave deterioro de las condiciones de vida. Señala que aunque hubo un fuerte aumento de las tasas de participación laboral entre 1979 y 1991 en México, se advierte un crecimiento pronunciado de trabajadores que laboró menos de 40 horas a la semana. Plantea que en la capital del país, durante el periodo de ajuste se registró un estancamiento en las horas semanales de trabajo entre 1987 a 1992. Por otra parte, la autora precisa que una parte del aumento del número de mujeres que participaron en el mercado laboral se debió en buena medida a los cambios que experimentó la demanda de mano de obra. En tanto aumentaron para las mujeres las oportunidades en el sector asalariado durante el periodo de estabilización y ajuste, la autora cuestiona la hipótesis de la inserción laboral femenina a partir de la baja del ingreso familiar.

\section{b) Enfoque de redes}

El enfoque de redes enfatiza el análisis de los vínculos que pueden establecerse entre distintos actores. Su importancia en relación a la reproducción de los grupos domésticos radica en que las redes pueden constituir uno de los recursos a los cuales éstos pueden recurrir frente a situaciones de crisis económica, en tanto pueden permitir el acceso a diversos tipos de bienes y servicios.

Un estudio ya clásico en el tema del acceso a recursos a partir de redes sociales es el de Larissa Lomnitz (1973), quien destaca como mecanismos de subsistencia de los grupos marginados en México, el establecimiento de redes de intercambio que permiten suplir la falta de seguridad económica. Dichas redes representarían un esquema de organización social adaptado a las necesidades de sobrevivencia de los sectores marginados socioeconómicamente. Frente a dichas necesidades, la autora demostró que los pobladores desarrollan redes de intercambio como un mecanismo efectivo para paliar la inseguridad económica, dando paso a una respuesta de tipo evolutivo al contexto de marginalidad (2001:48).

Lomnitz define las redes a partir del intercambio recíproco de bienes y servicios entre familias nucleares, en ese sentido representa un intercambio simétrico. En general se ha caracterizado estas redes por los intercambios y sistemas de reciprocidad, definido como “... un intercambio paritario de bienes y servicios como parte integral de una relación social duradera." (Polanyi, 1968 y Dalton, 1968 en Lomnitz, 1994).

El contenido de los intercambios en las redes han sido relevados por distintos autores (Lomnitz, 2001; Espinoza, 1995; De Oliveira y Salles, 1989) y están constituidos por: información; entrenamiento y ayuda para el empleo; préstamos de dinero; bienes compartidos en común; servicios; apoyo emocional y moral.

A este tipo de intercambio simétrico, Lomnitz agrega tres patrones de intercambios asimétricos que se da cuando una familia dentro de una red posee una mayor cantidad de recursos económicos: cuatismo, cacicazgo y familias en proceso de urbanización, lo cual conlleva un cambio en su nivel de vida; esta situación implica que la red se convierte en un estorbo para esta familia más que en un apoyo (Lomnitz, 2001:78). A estos tipos asimétricos se agrega una forma particular de intercambio: el intercambio 
diádico, el cual se puede desarrollar de un miembro a otro de grupos domésticos diferentes. $^{7}$

Otros autores también han destacado el papel que tienen las redes en la reproducción de los grupos domésticos. Se ha destacado el concepto de red de relaciones, para referirse a los diversos contactos establecidos dentro y entre los grupos domésticos basados en vínculos de intercambio y reciprocidad, los cuales son recursos fundamentales para la satisfacción de necesidades de la unidad doméstica (Roberts, 1973 en De Oliveira y Salles,1989: 19). En este contexto multirelacional, los vínculos establecidos entre diversos grupos domésticos, tienen un papel clave como mecanismo de reproducción cotidiana, en tanto permiten el intercambio de trabajo y de diversos tipos de favores (De Oliveira y Salles, 1989:20).

El papel de las redes no sólo ha sido vinculado a contextos de marginalidad, sino también a procesos de cambio social. Al respecto Chiarello (1994) señala la mayor intensidad en la construcción de redes sociales por parte de los grupos domésticos, a partir de los procesos de modernización social. Dicha red de relaciones representaría un papel importante en la organización familiar. Este entramado se constituiría por vecinos, amigos y asociaciones voluntarias.

Investigaciones realizadas en otros países de América Latina, también han relevado la importancia de las redes en las formas de sobrevivencia de las familias. Al respecto, Espinoza (1995) señala en un estudio realizado en Chile, cómo las redes permiten acceder a distintos recursos, en el ámbito del mercado de trabajo, intercambios monetarios, apoyo al hogar, cuidado de niños, tareas del hogar y construcción.

Si bien el estudio de las redes en su aporte a la sobrevivencia de los hogares ha sido analizado preponderantemente en contextos de pobreza urbana, otros trabajos han indagado en el papel de las redes en la reproducción de familias de distintos sectores socioeconómicos. Un estudio realizado en Chile (Márquez y Espinoza, 1997) que tuvo como objetivo identificar los modos en que las familias definen y enfrentan situaciones de inseguridad a lo largo de su historia, realizó entrevistas a miembros de familias de distintos niveles socioeconómicos y diversas zonas geográficas. Dicho trabajo se centró en lo que denominaron sociabilidad y redes sociales, las cuales establecen - según el estudio- un campo de oportunidades sobre el cual las familias desarrollan su acción. En términos socioeconómicos, la sociabilidad conecta y permite a las familias la obtención de recompensas sociales, a través de redes sociales laborales, de apoyo doméstico o de información. Desde el punto de vista normativo, las normas se acatan o esquivan con un alto grado de refuerzo de los círculos de relaciones familiares.

El estudio señala que las estrategias de respuesta a una situación de inseguridad familiar describen los comportamientos individuales y colectivos que se ponen en marcha para superar la crisis. La mayor parte de las historias realizadas, muestra precisamente que la familia por si sola, no puede proveer todos los mecanismos de integración. Los recursos que hacen eficaces las normas, que les permiten generar orden y permiten una movilidad socio-económica, muchas veces quedan al margen de los recursos internos de la familia; la estrategia más eficaz es la que pone en contacto al núcleo familiar con recursos externos; si la familia sólo cuenta con los recursos internos, éstas se enfrentan al riesgo del empobrecimiento (Márquez y Espinoza, 1997). 
El acceso a recompensas sociales, especialmente económicas, constituye otro ámbito externo de acción de las familias; las recompensas o recursos sociales no pertenecen a la vida cotidiana de las familias, sino que logran acceder a éstas de acuerdo a diversos mecanismos. De estos mecanismos, las redes sociales de las familias se cuentan como la base a partir de la cual se organiza la acción. "La sociabilidad no es un recurso entre otros, sino que constituye la propia estructura de oportunidades. Las propiedades de los lazos sociales son las que permiten a las familias establecer canales de acceso a recursos, pero no sólo eso, sino que actúan como una restricción insoslayable al establecimiento de estrategias. Las redes sociales y su manejo definen la sociabilidad de las familias; estas prácticas sociales son las que permiten transitar niveles de análisis desde la vida cotidiana a la organización social general." (Márquez y Espinoza, 1997: 42).

Con base en los estudios revisados, el enfoque de redes sociales puede analizar dichos vínculos como estrategias en sí mismas desarrolladas por parte de los hogares para acceder a distintos tipos de intercambio; también desde este enfoque, el análisis de redes puede evidenciar más bien la estructura de oportunidades o una dimensión de esta estructura, como un abanico de posibilidades con las que cuentan las familias para su reproducción y para el desarrollo de estrategias destinadas a ello.

\section{c) Enfoque vulnerabilidad-activos}

Dentro del ámbito de los estudios sobre la pobreza, recientemente se ha desarrollado un enfoque que ha incorporado nuevos elementos a su análisis y que involucra el estudio de las estrategias de los hogares. Caroline Moser $(1996,1998)$ ha desarrollado el enfoque de vulnerabilidad-activos ("asset vulnerability framework") y con base en éste, Kaztman y otros $(1999,2002)$ han planteado el enfoque activos-vulnerabilidadestructura de oportunidades (AVEO). Dichos enfoques se centran en lo que denominan la vulnerabilidad de los hogares e incorporan la noción de activos, capitales o recursos movilizados por parte de los hogares (Filgueira, 1999: 166). ${ }^{8}$

Moser (1996) señala que el concepto de vulnerabilidad alude a la inseguridad del bienestar de los individuos, los hogares o las comunidades ante un medioambiente cambiante; dichos cambios pueden ser ecológicos, económicos, sociales o políticos. El análisis de la vulnerabilidad - plantea-, identifica no sólo la amenaza, sino también la capacidad de adaptación en relación al aprovechamiento de las oportunidades y la resistencia a los efectos negativos del cambio. Los medios de resistencia son los activos que pueden movilizar los individuos, hogares o comunidades para hacer frente a las dificultades. En ese sentido la vulnerabilidad está estrechamente ligada a la posesión de activos.

El enfoque denominado de vulnerabilidad-activos, pone el acento en la dinámica de la formación de distintos tipos de capital potencialmente movilizables y las relaciones entre éstos; así como en los procesos en que hay pérdida, desgaste o factores limitantes para reponer el capital. El concepto de activos, por tanto, implica la posibilidad de observar los grados variables de posesión, control e influencia que los individuos tienen sobre los recursos y las diversas estrategias que desarrollan para movilizarlos (Filgueira, 1999:171). Las estrategias familiares, desde este enfoque, son 
parte de las acciones que pueden realizar los hogares para disminuir su vulnerabilidad; a través de la intensificación o diversificación de sus activos.

Los activos pueden ser tangibles e intangibles y se refieren a: mano de obra, capital humano, activos productivos, relaciones familiares y capital social. Los cambios del ambiente pueden erosionarlos o reforzarlos. La posibilidad de los hogares de evitar o reducir su vulnerabilidad y aumentar su productividad económica, dependen no sólo de sus activos iniciales, sino de su capacidad para transformar esos activos en ingresos, alimentos y otras necesidades básicas. Según Moser los activos pueden transformarse de dos maneras: mediante la intensificación de las estrategias existentes o a través de la creación y diversificación de otras estrategias.

La forma y eficacia del uso de activos y estrategias, dependerá de factores familiares y comunitarios. En términos de los hogares, incidirán características como su ciclo vital y las asimetrías en cuanto a derechos y obligaciones diferenciadas por sexo y edad. Con respecto a la comunidad, seguramente influirá en su capacidad de reacción ante los cambios el capital social, es decir, la confianza, las normas y redes de reciprocidad (Moser, 1996:3).

A partir del estudio de cuatro comunidades pobres en distintos países (Zambia, Ecuador, Filipinas, Hungría), que sufrieron en la década de los ochenta dificultades económicas, Moser encontró distintas estrategias que desarrollaron los hogares para hacer frente a las dificultades económicas, a través de la movilización de sus activos; y distintos elementos que pueden afectar negativamente dichas estrategias. ${ }^{9}$ Como veremos, tienen elementos comunes con algunas estrategias encontradas en los estudios analizados en el apartado precedente:

a) La mano de obra como un activo. En el estudio se encuentra que con frecuencia los hogares movilizan mano de obra adicional ante la escasez de ingresos, principalmente a través de la incorporación de mujeres -y niños en hogares más pobres- al trabajo.

b) La infraestructura social y económica como un activo. La falta de servicios públicos - agravada en la década de los ochenta y noventa- ha repercutido en la capacidad de los hogares pobres para generar ingresos. A medida que la inversión pública ha disminuido, los hogares han debido destinar recursos para reemplazarlos por servicios privados (salud, por ejemplo); a prescindir de ellos o buscar formas de acceder a los mismos (acarreo de agua). Esto lleva a un aumento por parte de las mujeres del tiempo dedicado a la obtención de servicios.

c) La vivienda como un activo. Frente a la crisis económica, los hogares pobres pueden utilizar sus viviendas para instalar alguna empresa o rentarla. En caso extremo pueden vender sus activos. También utilizan la vivienda para ampliar sus relaciones personales y generar capital social. La posibilidad de éxito de las empresas, sin embargo, depende de activos complementarios como electricidad, agua, conocimientos especializados, crédito.

d) Las relaciones familiares como un activo. En el estudio se detectaron cambios en la estructura y composición de los hogares con el fin de establecer redes de apoyo familiar. La autora señala que dichos cambios son tanto productos de la vulnerabilidad como una estrategia para reducirla. Como tendencias comunes en los cuatro casos se 
detectó: aumento de hogares encabezados por mujeres; pobreza mayormente en hogares extensos; aumento de familias extensas para dar refugio y proveer de seguridad a individuos vulnerables $\mathrm{y} / \mathrm{o}$ con el objeto de eficientar los recursos como alimentos, ingreso, cuidado de niños. Asimismo se detectó el incremento de migración laboral y remesas.

e) El capital social como un activo. En este punto se establecen paradojas ante la crisis. Por una parte la situación de crisis económica puede reforzar el capital social normas, confianza y redes de reciprocidad-. Por otro lado puede darse un menoscabo del mismo en tanto se produce una pérdida de confianza en la comunidad.

El capital social de una comunidad constituye un importante activo gracias al cual disminuye la vulnerabilidad y aumentan las oportunidades, a través del préstamo de dinero entre conocidos o del apoyo en el cuidado de niños, por ejemplo. También a través de la mayor organización comunitaria para resolver la provisión de servicios urbanos. Sin embargo, cuando las comunidades se empobrecen, su reserva de capital social puede erosionarse. Los hogares que llegan a un alto nivel de erosión de sus activos, tienden a concentrarse en su propia supervivencia y por tanto se resienten los vínculos comunitarios. A la vez ante la caída de los ingresos puede aumentar la violencia, con la consiguiente pérdida de confianza en la comunidad.

Un aporte importante de este enfoque es que rescata los efectos negativos que pueden conllevar la implementación de algunas estrategias al interior de los hogares. En su estudio Moser señala que las estrategias para reducir la vulnerabilidad a veces imponen una carga desigual a los distintos miembros del hogar. Las mujeres, por la multiplicidad de responsabilidades que asumen, tienen una carga desproporcionada en el proceso de adaptación a las dificultades económicas. ${ }^{10}$

Otra contribución interesante, es el análisis que se hace en relación a la dinámica interna de los hogares con el entorno institucional. Dicha cuestión es retomada más decididamente por el enfoque de AVEO desarrollado por Kaztman y sus colaboradores (1999, 2002).

El enfoque AVEO introduce el concepto de estructura de oportunidades, las cuales se definen como las probabilidades de acceso a bienes, servicios o desempeño de actividades. Dichas oportunidades inciden en el hogar en la medida que permiten o facilitan a sus miembros el uso de sus propios recursos o les proveen de nuevos. En dicho planteo las tres fuentes centrales de oportunidades son el mercado, el estado y la sociedad. El análisis se centra entonces en la relación establecida entre las estrategias de los hogares y la estructura de oportunidades existente.

Un aspecto importante es la distinción que se establece entre recursos y activos. Los primeros refieren a los bienes tangibles e intangibles que controla un hogar. Los segundos, por su parte, aluden al subconjunto de esos recursos cuya movilización permite el aprovechamiento de las estructuras de oportunidades existentes en un momento, ya sea para elevar el nivel de bienestar o mantenerlo ante situaciones que lo amenazan (Kaztman y Filgueira, 1999:31).

Una cuestión interesante de dichos estudios es la combinación de metodologías y técnicas para analizar el tema de las estrategias, la movilización de activos y la 
estructura de oportunidades. Al respecto, uno de los estudios incluido en la investigación sobre vulnerabilidad social en Uruguay en 1999, busca profundizar en el conocimiento de las estrategias de sobrevivencia y el mejoramiento de las condiciones y oportunidades de bienestar de hogares urbanos en condiciones de vulnerabilidad. Para ello realiza entrevistas en profundidad a 53 familias que han enfrentado o enfrentan situaciones críticas. La selección de casos fue intencional, de acuerdo a los objetivos del estudio, priorizando por hogares en etapa de formación (con crianza de hijos) (Zaffaroni, 1999)

Dentro de los recursos movilizados por las familias destacan el trabajo; el capital humano y el capital social, además de los recursos materiales. En la generación de dichos recursos inciden factores relacionados con la propia familia; con el mercado, la comunidad y los servicios a los cuales acceden. Dentro de las estrategias que destacan, advierten dos tipos principales: aquellas destinadas a obtener una mejora en sus niveles de ingreso y bienestar; y otras que implican sólo una adaptación momentánea, que llevan a un deterioro y pérdida en relación a sus anteriores condiciones de trabajo y calidad de vida (Zaffaroni, 1999:63-65). En este sentido es interesante la diferenciación que hacen Kaztman y Filgueira entre estrategias de promoción, destinadas a mejorar la situación de bienestar presente y estrategias de adaptación, destinadas a mantener el bienestar y evitar su deterioro (1999: 32).

Otro estudio que forma parte del trabajo ya citado se centró en la exploración de las potencialidades de aplicación de las Encuestas de Hogares y las Encuestas de Ingresos y Gastos de ese país para el análisis de los activos y recursos movilizados por éstos. Para ello se orientó a la exploración de indicadores que permitieran hacer observables algunos activos, los cuales fueron seleccionados a partir de tres áreas prioritarias: mercado, estado y sociedad (Filgueira, 1999).

Como parte de los indicadores seleccionados para indagar sobre el ámbito del mercado, se establecieron aquellos relacionados con el trabajo de los distintos miembros de la familia. En cuanto a aquellos indicadores delimitados para establecer los activos desde el estado, se orientaron a las temáticas de protección y seguridad social. Finalmente, los indicadores de capital social, se orientaron a analizar aspectos de uso del capital social; existencia del mismo y capital social familiar.

d) Enfoque de curso de vida

El análisis de las estrategias desde la perspectiva del curso de vida deriva principalmente de estudios realizados desde la demografía o la historia (Moen y Wethington, 1992; Hareven, 2000). En este enfoque la noción de estrategias familiares combina aspectos de las teorías estructurales y de elección racional en un marco temporal, situando a las estrategias familiares en un amplio contexto histórico, social y cultural que cambia las oportunidades y constreñimientos, recursos y demandas, normas y expectativas. Las transformaciones a lo largo del tiempo en los ambientes social, institucional y económico, produce fluctuaciones en los recursos, necesidades y aspiraciones familiares.

La formulación del curso de vida además enfatiza la temporalidad natural de las estrategias familiares. Según este enfoque las familias son capaces de moverse en varias opciones a lo largo del ciclo vital y son diferencialmente capaces de movilizarse 
en respuesta a las exigencias externas, a partir de los recursos disponibles y las interpretaciones subjetivas de sus miembros.

Se establece entonces una relación entre estrategias familiares y cambio social. Los individuos y familias hacen elecciones enfrentando recursos y constricciones; sus elecciones devienen fuerzas causales que modelan futuros recursos y constricciones y que contribuyen a formar tendencias y patrones de cambio social en el tiempo. El enfoque distingue cómo los distintos miembros de la familia cumplen múltiples roles a lo largo de su curso de vida en su relación con los cambios (jefe/a de familia; cónyuges, hijos, parientes y no parientes). En dicho contexto, el enfoque diferencia entre estrategias de corto, mediano y largo plazo.

Desde este marco, Hareven realizó un estudio sobre estrategias familiares en un contexto de cambio histórico, económico y social. Analizó las estrategias familiares en relación al proceso de industrialización en la sociedad norteamericana durante el siglo $X I X$, en una ciudad industrial. A partir de este caso, identificó una serie de estrategias desarrolladas por los inmigrantes que se insertaban en el trabajo industrial, donde el parentesco jugaba un importante papel:

- Apoyo familiar para encontrar trabajo.

- Apoyo familiar para la asistencia y sobrevivencia.

- Participación de niños y mujeres en el mercado laboral.

- Incorporación de huéspedes en el hogar.

- Cambio en los patrones de consumo familiar.

- Cambios en la composición familiar.

Como se advierte, más allá de la temporalidad y espacialidad diferente, muchas estrategias familiares se repiten ante situaciones de cambio e incertidumbre. 


\begin{tabular}{|c|c|c|}
\hline \multicolumn{3}{|c|}{ RECURSOS Y ESTRATEGI AS DESPLEGADAS POR LOS HOGARES SEGÚN DI STI NTOS AUTORES } \\
\hline Autor & Recursos/ Activos & Estrategias \\
\hline $\begin{array}{l}\text { M. González de } \\
\text { la Rocha (1990) }\end{array}$ & & $\begin{array}{l}\text { Intensificación del trabajo asalariado y doméstico } \\
\text { Reacomodo en la división del trabajo } \\
\text { Cambios en el tamaño y composición del hogar } \\
\text { Cambios en la estructura social del hogar } \\
\text { Cierta modificación en los patrones de consumo }\end{array}$ \\
\hline $\begin{array}{l}\text { Selby et. Al. } \\
(1990,1994)\end{array}$ & & $\begin{array}{l}\text { Descenso de la tasa de natalidad } \\
\text { Disminución de los miembros del hogar } \\
\text { Complejización de los hogares }\end{array}$ \\
\hline $\begin{array}{l}\text { G. A. Cornia } \\
\text { (1987) }\end{array}$ & & $\begin{array}{l}\text { Estrategias destinadas a la generación de recursos: Incremento de la } \\
\text { oferta de fuerza de trabajo en la economía; incremento de la producción } \\
\text { propia; cambios en la posición de activos-pasivos; aumento del flujo de } \\
\text { ingresos por transferencia. } \\
\text { Estrategias para mejorar la eficacia de los recursos existentes: cambios } \\
\text { en los hábitos de compra; cambios en los hábitos de preparación de } \\
\text { alimentos; cambios en las pautas globales de consumo; cambios en las } \\
\text { pautas dietéticas; cambios en la distribución intra-familiar de alimentos. } \\
\text { Estrategias de familia extendida y de migración: cambios en la } \\
\text { organización y composición de la familia; emigración. }\end{array}$ \\
\hline \multirow{5}{*}{$\begin{array}{l}\text { Moser, C. (1996, } \\
\text { 1998) }\end{array}$} & Trabajo & $\begin{array}{l}\text { Aumento de miembros de la familia que trabajan, especialmente mujeres. } \\
\text { Aumento de industrias caseras. } \\
\text { Aumento del número de empleos por trabajador }\end{array}$ \\
\hline & $\begin{array}{l}\text { Capital humano/ } \\
\text { Infraestructura } \\
\text { social y económica }\end{array}$ & $\begin{array}{l}\text { Sustitución de servicios públicos por privados como bombas de agua, } \\
\text { atención de la salud y educación } \\
\text { Modificación de patrones de consumo: cambio de hábitos alimenticios; } \\
\text { supresión de compras de algunos bienes. }\end{array}$ \\
\hline & $\begin{array}{l}\text { Activos productivos: } \\
\text { Vivienda }\end{array}$ & $\begin{array}{l}\text { Solución del problema de inseguridad de la tenencia. } \\
\text { Uso del terreno para residencias intergeneracionales. }\end{array}$ \\
\hline & $\begin{array}{l}\text { Relaciones } \\
\text { familiares }\end{array}$ & $\begin{array}{l}\text { Aumento de familias extensas } \\
\text { Aumento de migración laboral } \\
\text { Aumento de apoyo informal de los hogares }\end{array}$ \\
\hline & Capital social & $\begin{array}{l}\text { Acceso a créditos informales } \\
\text { Reciprocidad entre hogares } \\
\text { Mayor participación en actividades comunitarias }\end{array}$ \\
\hline \multirow{6}{*}{ Zaffaroni (1999) } & Trabajo & $\begin{array}{l}\text { Incremento de las horas de trabajo del jefe de hogar } \\
\text { Acceso a puesto de trabajo en mejores condiciones } \\
\text { Incorporación o reintegro de la mujer al mercado de trabajo } \\
\text { Trabajo infantil } \\
\text { Aceptación de condiciones desventajosas de trabajo } \\
\text { Pasaje a trabajador por cuenta propia }\end{array}$ \\
\hline & Capital humano & Adquisición y uso de formación \\
\hline & Vivienda & $\begin{array}{l}\text { Adquisición o autoconstrucción de vivienda } \\
\text { Uso de la vivienda para fines productivos }\end{array}$ \\
\hline & Lugar de radicación & Cambio de lugar de radicación (migración) \\
\hline & Ingresos & $\begin{array}{l}\text { Cambio en pautas de consumo } \\
\text { Uso del crédito } \\
\text { Venta de bienes } \\
\text { Endeudamiento }\end{array}$ \\
\hline & Capital social & $\begin{array}{l}\text { Vinculaciones con familiares directos y vecinos cercanos } \\
\text { Participación en organizaciones } \\
\text { Apoyo en la cohesión familiar }\end{array}$ \\
\hline
\end{tabular}

Fuente: Elaboración propia a partir de los autores. La sistematización de Moser está basada en sus propios cuadros 


\section{Comentarios finales}

Como se advierte, los estudios de estrategias familiares, han aportado con conocimiento cualitativo y cuantitativo acerca de las diversas actividades y acciones que las familias e individuos en condiciones de pobreza despliegan en la región, con el objeto de reproducirse y vivir cotidianamente. Sin embargo, quedan aún interrogantes que sería importante abordar en las nuevas investigaciones que se realicen en torno a este objeto de estudio. Una de ellas dice relación con la posibilidad de ampliar el estudio de estas prácticas a diversos sectores sociales. Si bien ha habido diversidad de estudios en sectores pobres, fundamentado - entre otros aspectos- en el impacto de las crisis económicas, es posible pensar en el interés de analizar las estrategias que desarrollan sectores medios, por ejemplo, frente a los cambios y crisis económicas que los han afectado en las últimas décadas.

Asimismo, se plantea como algo imprescindible, incorporar variables que complejicen el análisis, como las dimensiones étnicas, de género y generación. Así como la pobreza afecta diferencialmente a la población según sus características sociodemográficas y las construcciones culturales que se desarrollan en torno a ellas; las acciones que los individuos pueden desplegar frente a las inestabilidades y crisis, también se presentan de manera diferenciada y desigual.

Por otra parte, sería deseable que los estudios pudiesen derivar en insumos y recomendaciones para la implementación de políticas sociales destinadas a la población en condiciones de pobreza y que, desde el estado, se consideren dichos hallazgos para la corrección de dichas iniciativas.

Finalmente, pensamos que falta ahondar mayormente en las condicionantes individuales y estructurales que inciden en el éxito o fracaso de determinadas iniciativas estatales y prácticas individuales y colectivas que desarrollan los sujetos para la reproducción. A partir del análisis del caso mexicano, por ejemplo -como se ha visto para otros casos-, se detectó que ciertas iniciativas de políticas dirigidas a mujeres para la generación de ahorro, incentivaban mayormente el aislamiento y la diferenciación social dentro de la localidad en que operaban dichas iniciativas. RM

\section{Bibliografía}

ARGÜELLO, OMAR, Estrategias de supervivencia: un concepto en busca de su contenido, en Demografía y Economía, vol. 2, pags.: 190-203, Colegio de México.

ARRIAGADA, IRMA, 2007, Familias y políticas públicas en América Latina, CEPAL, Santiago de Chile.

BARSOTTI, CARLOS, La organización social de la reproducción de los agentes sociales, las unidades familiares y sus estrategias, en Demografía y Economía, vol. 2, pags.: 164189, Colegio de México.

CORNIA, GIOVANNI ANDREA, 1987, "Adjustement at the Household Level: Potentials and Limitations or Survival Strategies", en Cornia et. Al., (eds) Adjustment with a Human Face, Protecting the Vulnerable and Promoting Growth, Vol I Claredon Press, Oxford. 
CORROCHANO, DAVID, 2003, "El capital social como problema”, (inédito).

CHIARELLO, FRANCO, 1994, "Economía informal, familia y redes sociales", en René Millán (comp.), Solidaridad y producción informal de recursos, Instituto de Investigaciones Sociales, UNAM, Porrúa, México

DAMIÁN, ARACELI, 2002, Cargando el ajuste: los pobres y el mercado de trabajo en México, El Colegio de México.

DE OLIVEIRA, ORLANDINA Y VANIA SALLES, "Acerca del estudio de los grupos domésticos: un enfoque sociodemográfico", en: Orlandina de Oliveira et. Al. (comp..), Grupos domésticos y reproducción cotidiana, UNAM, COLMEX, Porrúa, México, 1989.

DUHAU, EMILIO, "Gobernabilidad democrática a nivel local. Problemas de la democracia participativa como alternativa al clientelismo y el corporativismo", en Miguel Bazdresch (coord..), Memoria del Congreso. Gobiernos Locales: el futuro político de México, ITESO, México, 2000.

ESPINOZA, VICENTE, 1995, Redes sociales y superación de la pobreza, En: Revista de Trabajo Social, No 66, Santiago de Chile.

ESPINOZA VICENTE Y FRANCISCA MÁRQUEZ, 1997, "Vivir la inseguridad en Chile: cotidianidad y trayectoria de familia", Documentos de Trabajo, SUR, Santiago de Chile.

FILGUEIRA, CARLOS H., 1999, "Vulnerabilidad, activos y recursos de los hogares: una exploración de indicadores", en: Kaztman, Rubén, Activos y estructuras de oportunidades. Estudio sobre las raíces de la vulnerabilidad social en Uruguay, PNUD, CEPAL, Uruguay.

GARCÍA, BRÍGIDA Y ORLANDINA DE OLIVEIRA, 1994, Trabajo femenino y vida familiar en México. El Colegio de México, 1994.

GARRIDO MEDINA, LUIS Y ENRIQUE GIL CALVO, 1993, "El concepto de estrategias familiares", en Luisa Garrido Medina y Enrique Gil Calvo (eds.), Estrategias familiares, editorial Alianza, Madrid.

GONZÁLEZ DE LA ROCHA, MERCEDES, 1986, Los recursos de la pobreza. Familia de bajos ingresos en Guadalajara. El Colegio de Jalisco.

GONZÁLEZ DE LA ROCHA, MERCEDES, 1991, "Family well-being, food consumption, and survival strategies during Mexico's economic crisis", en Mercedes González de la Rocha y Agustín Escobar Latapí, Social responses to Mexico's economic crisis of the 1980's, Center for U.S.-Mexican Studies, University of California, San Diego.

GONZÁLEZ DE LA ROCHA, MERCEDES; AGUSTÍN ESCOBAR Y MARÍA DE LA O MARTÍNEZ CASTELLANOS, 1990, "Estrategia versus conflicto: reflexiones para el estudio del grupo doméstico en época de crisis", en Guillermo de la Peña et. Al. (compiladores), Crisis, conflicto y sobrevivencia. Universidad de Guadalajara, CIESAS, México. 
HAREVEN, TAMARA, 2000, Families, history and social change, Westview Press.

KAZTMAN, RUBÉN, 1999, Activos y estructuras de oportunidades. Estudio sobre las raíces de la vulnerabilidad social en Uruguay, PNUD, CEPAL, Uruguay.

KAZTMAN, RUBÉN; GUILLERMO WORMALD (coordinadores), 2002, Trabajo y ciudadanía. Los cambiantes rostros de la integración y exclusión social en cuatro áreas metropolitanas de América Latina. Uruguay.

LOMNITZ, LARISSA,1994, Redes sociales, cultura y poder, FLACSO, Porrúa, México.

MEERT, Rural community and the importance of reciprocal survival strategies, 2000, Sociologia Ruralis, vol. 40, num. 3, pags: 319-338, Julio.

MOEN, PHYLLIS Y ELAINE WETHINGTON, 1992, The concept of family adaptative strategies, Annual Review of Sociology, vol. 18, pags: 233-251.

MOSER, CAROLINE O.N., 1996, "Situaciones críticas. Reacción de las familias de cuatro comunidades urbanas pobres ante la vulnerabilidad y la pobreza.", ESD, Serie de estudios y monografías sobre el desarrollo ecológicamente sostenible, No 75.

MOSER, CAROLINE O.N., 1998, The Asset Vulnerability Framework: Reassesing Urban Poverty Reduction Strategies, en World Development, vol. 26, No.1, pags: 1-19.

SELBY, HENRY, ET. AL., 1990, "La familia urbana mexicana frente a la crisis", en Guillermo de la Peña et. Al. (compiladores), Crisis, conflicto y sobrevivencia. Universidad de Guadalajara, CIESAS, México.

SELBY, HENRY, ET. AL., 1994, La familia en el México urbano. Mecanismos de defensa frente a la crisis (1978-1992). CONACULTA, México.

TUIRÁN, RODOLFO, 1993, "Estrategias familiares de vida en época de crisis", CEPAL, Santiago de Chile.

ZAFFARONI, CECILIA, 1999, "Los recursos de las familias urbanas de bajos ingresos para enfrentar situaciones críticas", en Katzman, Rubén (coordinador), Activos y estructuras de oportunidades. Estudio sobre las raíces de la vulnerabilidad social en Uruguay, PNUD, CEPAL, Uruguay.

\section{Notas}

1 Este trabajo es parte de mi tesis doctoral Prácticas familiares de reproducción en contextos de cambio.
Milpa Alta, Distrito Federal, México, UNAM, 2007. En este sentido, si bien el artículo reflexiona en el contexto
de Latinoamérica, los principales referentes empíricos derivan del caso mexicano. Reconocemos que existe
una mayor cantidad de estudios sobre la temática que nos ocupa, sin embargo, además de la imposibilidad
temporal de abarcar la totalidad del conjunto, pensamos que la selección realizada representa las principales
líneas desarrolladas en este campo. Este trabajo es parte de mi tesis doctoral Prácticas familiares de

Revista Mad. $N^{\circ}$ 17, Septiembre de 2007. Departamento de Antropología. Universidad de Chile http://www.revistamad.uchile.cl/17/arteaga_07.pdf 
reproducción en contextos de cambio. Milpa Alta, Distrito Federal, México, 2007. En este sentido, si bien el artículo reflexiona en el contexto de Latinoamérica, los principales referentes empíricos derivan del caso mexicano.

${ }^{2}$ Según datos de la CEPAL para 2006, el 36.5\% de la población de la región se encuentra en condiciones de pobreza. Chile contaría con un $\mathbf{1 3 . 7 \%}$ de población con estas características y México con un $31.7 \%$.

${ }^{3}$ Arriagada (2007) señala - entre otros- que a nivel de la región latinoamericana se ha producido entre 1990 y 2005 una reducción de las familias nucleares; aumento de hogares monoparentales con jefas y aumento de hogares no familiares.

${ }^{4}$ Bajo este enfoque se ha desarrollado el estudio de distintos comportamientos de los grupos domésticos para su reproducción, los cuales se engloban bajo distintos conceptos: estrategias de sobrevivencia, estrategias de existencia, estrategias familiares, estrategias de supervivencia, estrategias familiares de vida - entre otros-. Para una revisión del debate al respecto véase Revista Demografía y Economía, vol.2, 1981.

${ }^{5}$ Parte del debate teórico y metodológico en torno al concepto de estrategias familiares se plasmó en la revista Demografía y Economía de 1981, vol. 2. En sus artículos se encuentran recogidas varias cuestiones retomadas en el presente texto.

${ }^{6}$ En el texto citado de 1990, la autora habla de "respuesta racional-colectiva" ante la crisis económica.

7 A este tipo de intercambios, puede agregarse el clientelismo, que implica una relación entre patrón y cliente; en su versión moderna, se trata principalmente de un intercambio de votos por favores sustentado en un gran pragmatismo (Corrochano, 2002:138). Como señala Duhau (2000), "El clientelismo urbano, lejos de responder a prácticas 'tradicionales', emerge y se reproduce en condiciones de acelerada urbanización. Consiste en prácticas sociales y en formas de relación tejidas entre grupos determinados de la población (predominante pero no exclusivamente pertenecientes a los sectores populares) y políticos o autoridades, regidas por la lógica del intercambio y mediadas por un tercero que opera como intermediario (líder, dirigente, etc...)".

${ }^{8}$ Por activos se hace referencia al conjunto de recursos materiales e inmateriales que los individuos y hogares movilizan para mejorar su desempeño económico y social o para evitar el deterioro de sus condiciones de vida o disminuir su vulnerabilidad (Filgueira, 1999: 166).

${ }^{9}$ La metodología se centró en muestreos aleatorios de 200 hogares por comunidad, a los cuales se aplicó la técnica del estudio de panel.

10 En el trabajo ya citado de González de la Rocha (1990), a partir de una crítica al enfoque de las estrategias de sobrevivencia, señala la falta de identificación del conflicto que producen precisamente dichas estrategias, lo cual se refleja, por ejemplo, en el aumento de la violencia intradoméstica. 